PROCEEDINGS OF THE

AMERICAN MATHEMATICAL SOCIETY

Volume 131, Number 7, Pages 2145-2153

S 0002-9939(02)06838-7

Article electronically published on December 30, 2002

\title{
TYPE III FACTORS ARISING FROM CUNTZ-KRIEGER ALGEBRAS
}

\author{
RUI OKAYASU
}

(Communicated by David R. Larson)

\begin{abstract}
We determine the types of factors arising from GNS-representations of quasi-free KMS states on Cuntz-Krieger algebras. Applying our result to the Cuntz-Krieger algebras arising from the boundary actions of some amalgamated free product groups, we also determine the types of harmonic measures on the boundaries.
\end{abstract}

\section{INTRODUCTION}

The Cuntz algebra $\mathcal{O}_{n} \mathrm{Cun}$ and the Cuntz-Krieger algebra $\mathcal{O}_{A}[\mathrm{CK}$, a generalization of $\mathcal{O}_{n}$, are important examples of $C^{*}$-algebras. The Cuntz-Krieger algebra $\mathcal{O}_{A}$, associated with a 0-1 matrix $A$, is the universal $C^{*}$-algebra generated by the family of partial isometries $\left\{S_{i}\right\}_{i=1}^{N}$ satisfying the Cuntz-Krieger relations. The universal property of $\mathcal{O}_{A}$ allows us to define the so-called gauge action on $\mathcal{O}_{A}$. The existence of KMS states for one-parameter automorphisms is one of the natural questions. The KMS states for the gauge actions on $\mathcal{O}_{n}$ and $\mathcal{O}_{A}$ were obtained by D. Olesen and G. K. Pedersen [OP], and M. Enomoto, M. Fujii and Y. Watatani [EFW], respectively. More generally, D. E. Evans determined the KMS states on $\mathcal{O}_{n}$ for the quasi-free actions in Eva. In order to construct examples of subfactors, M. Izumi determined the types of factors obtained by the GNS-representations of quasi-free KMS states in [Izu]. One of the purposes of this paper is to generalize his result to Cuntz-Krieger algebras. The existence and the uniqueness of quasi-free KMS states on Cuntz-Krieger algebras were proved by R. Exel and M. Laca in [EL]. It implies that the von Neumann algebras arising from their GNS-representations are factors. We will compute the Connes spectrum of the modular automorphism group and determine the types of quasi-free KMS states.

As an application, we can give a construction of type III factors from geometric objects. J. Spielberg proved in Spi] that some Cuntz-Krieger algebras can be obtained by the crossed product construction of the boundary action $(\partial \Gamma, \Gamma)$, where $\Gamma$ is the free product of cyclic groups and $\partial \Gamma$ is the hyperbolic boundary as a hyperbolic group. This construction was generalized to amalgamated free product groups in Oka. Under this identification, it was shown that there is one-to-one correspondence between quasi-free KMS states and some class of random walks on $\Gamma$; namely, by identifying $\partial \Gamma$ with the Poisson boundary, harmonic measures on $\partial \Gamma$ induce quasi-free KMS states. We will apply the main result to the harmonic

Received by the editors September 27, 2001 and, in revised form, February 19, 2002.

2000 Mathematics Subject Classification. Primary 46L30. 
measures and determine their types. It turns out that the resulting factors are either of type $\mathrm{III}_{1}$ or of type $\mathrm{III}_{\lambda}(0<\lambda<1)$, where $\lambda$ is some algebraic number. Therefore, by combining these results, we can make type III factors from boundary actions and harmonic measures on the boundary, which generalizes J. Ramagge and G. Robertson's result in $\mathrm{RR}$.

\section{Preliminaries}

2.1. Perron-Frobenius theorem. Let $A=[A(i, j)]_{i, j=1}^{N}$ be an $N \times N$ matrix with non-negative entries. We denote the $(i, j)$-entry of $A^{m}$ by $A^{m}(i, j)$. A matrix $A$ is irreducible if for every pair of indices $i$ and $j$ there is an $m>0$ with $A^{m}(i, j)>0$. For $1 \leq i, j \leq N$, put $E(i, j)=\left\{m \in \mathbb{N} \mid A^{m}(i, j)>0\right\}$ and $p(i)=$ g.c.d. $\{m \in$ $\left.\mathbb{N} \mid A^{m}(i, i) \neq 0\right\}$. Note that if $A$ is irreducible, then $p \equiv p(i)$ for any $i$ and we call it the period of $A$. An irreducible matrix $A$ is said to be periodic of period $p$ if $p>1$ and aperiodic if $p=1$. Set $I_{k}=\{i \mid 1 \leq i \leq N, E(i, 1)=k-1(\bmod p)\}$ for $k=1, \ldots, p$. If $A$ is periodic, then the index set $\{1, \ldots, N\}$ can be decomposed into distinct subsets $I_{1}, \ldots, I_{p}$ such that the matrix $A$ translates from $I_{k}$ into $I_{k+1}\left(I_{p}\right.$ into $I_{1}$ ), and the restriction of $A^{p}$ to $I_{k}$ is aperiodic. If $A$ is irreducible, the PerronFrobenius theorem guarantees the existence of the strictly positive eigenvector with respect to the simple root $\alpha$ of the characteristic polynomial such that $\alpha \geq|\beta|$ for any other eigenvalue $\beta$. Moreover, the following theorem is known.

Theorem 2.1 ([Kit, Theorem 1.3.8]). Let $A$ be an irreducible matrix with nonnegative entries and $p$ the period of $A$. If $x={ }^{T}\left(x_{1}, \ldots, x_{N}\right)$ and $y=\left(y_{1}, \ldots, y_{N}\right)$ are the right and left Perron eigenvectors of the Perron eigenvalue $\alpha$ such that $\sum_{i=1}^{N} x_{i} y_{i}=p$, then

$$
\lim _{n \rightarrow \infty} A^{p n}(i, j) / \alpha^{p n}=x_{i} y_{j},
$$

for any $i, j=1, \ldots, N$.

2.2. Cuntz-Krieger algebras. Let $A$ be an $N \times N$ - 1 matrix without zero rows. Then the Cuntz-Krieger algebra $\mathcal{O}_{A}$ is the universal $C^{*}$-algebra generated by the family of partial isometries $S_{1}, \ldots, S_{N}$ satisfying

$$
S_{i}^{*} S_{i}=\sum_{j=1}^{N} A(i, j) S_{j} S_{j}^{*} \quad \text { and } \quad 1=\sum_{i=1}^{N} S_{i} S_{i}^{*} .
$$

For $i=1, \ldots, N$, let us denote the initial projection of $S_{i}$ by $Q_{i}$ and the range projection by $P_{i}$. We say that $\xi=\left(\xi_{1}, \ldots, \xi_{n}\right) \in \prod_{i=1}^{n}\{1, \ldots, N\}$ with $A\left(\xi_{i}, \xi_{i+1}\right) \neq$ 0 is an admissible word and denote the set of all admissible words by $\mathcal{W}_{A}$. We define two maps $s$ and $r$ by $s(\xi)=\xi_{1}$ and $r(\xi)=\xi_{n}$. For $\xi=\left(\xi_{1}, \ldots, \xi_{n}\right), \eta=$ $\left(\eta_{1}, \ldots, \eta_{m}\right) \in \mathcal{W}_{A}$ with $A\left(\xi_{n}, \eta_{1}\right)=1$, we define the concatenation $\xi \cdot \eta$ in $\mathcal{W}_{A}$ by $\left(\xi_{1}, \ldots, \xi_{n}, \eta_{1}, \ldots, \eta_{m}\right)$. Let us say that an admissible word $\xi=\left(\xi_{1}, \ldots, \xi_{n}\right)$ is a loop if $A\left(\xi_{n}, \xi_{1}\right)=1$. We say that a loop $\xi$ is a circle if $\xi_{k} \neq \xi_{l}$ for any $1 \leq k, l \leq n$ $(k \neq l)$.

Let $\omega=\left(\omega_{1}, \ldots, \omega_{N}\right) \in \mathbb{R}_{+}^{N}$. We define the action $\alpha^{\omega}$ of $\mathbb{R}$ on $\mathcal{O}_{A}$ by $\alpha_{t}^{\omega}\left(S_{i}\right)=$ $e^{\sqrt{-1} \omega_{i} t} S_{i}$ for $t \in \mathbb{R}$ and $i=1, \ldots, N$. If $\omega=(1, \ldots, 1)$, then $\alpha^{\omega}$ is the gauge action. We define two word-length functions. For $\xi=\left(\xi_{1}, \ldots, \xi_{n}\right) \in \mathcal{W}_{A}$, we denote the canonical one by $|\xi|=n$ and the other by $\omega_{\xi}=\omega_{\xi_{1}}+\cdots+\omega_{\xi_{n}}$. Let $\Omega_{A}=\left\{\left(a_{k}\right)_{i=k}^{\infty} \mid A\left(a_{k}, a_{k+1}\right)=1\right\}$ be the set of all one-sided infinite admissible words. Note that there is the faithful conditional expectation $\Phi$ from $\mathcal{O}_{A}$ onto 
$\overline{\operatorname{span}}\left\{S_{\xi} S_{\xi}^{*} \mid \xi \in \mathcal{W}_{A}\right\} \simeq C\left(\Omega_{A}\right)$ (see [CK]). We assume that there is $\beta \in \mathbb{R}_{+}$and $x_{i}>0$ that satisfies

$$
x_{i}=\sum_{j=1}^{N} e^{-\beta \omega_{i}} A(i, j) x_{j} \quad \text { and } \quad 1=x_{1}+\cdots+x_{N} .
$$

We can define a probability measure $\nu$ on $\Omega_{A}$ by

$$
\nu\left(\Omega_{A}\left(\xi_{1}, \ldots, \xi_{n-1}, \xi_{n}\right)\right)=e^{-\beta \omega_{\xi_{1}}} \cdots e^{-\beta \omega_{\xi_{n-1}}} x_{\xi_{n}},
$$

where $\Omega_{A}\left(\xi_{1}, \ldots, \xi_{n}\right)$ is the cylinder set $\left\{\left(a_{k}\right)_{k=1}^{\infty} \in \Omega_{A} \mid a_{1}=\xi_{1}, \ldots, a_{n}=\xi_{n}\right\}$. This probability measure induces a $\beta$-KMS state for $\alpha^{\omega}$ on $\mathcal{O}_{A}$ by $\phi^{\omega}=\nu \circ \Phi$. Set $A_{\omega}(i, j)=e^{-\beta \omega_{i}} A(i, j)$. Note that the vector $x={ }^{T}\left(x_{1}, \ldots, x_{N}\right)$ is the right Perron eigenvector of the matrix $A_{\omega}$ with respect to the Perron eigenvalue 1. R. Exel and M. Laca, in fact, showed the following in [EL].

Theorem 2.2 (EL, Theorem 18.5]). If A is irreducible, then there exists the unique $\beta$-KMS state $\phi^{\omega}$ of the Cuntz-Krieger algebra $\mathcal{O}_{A}$ for the action $\alpha^{\omega}$, and the inverse temperature $\beta$ is also unique.

Throughout this paper, we assume that $A$ is irreducible and not a permutation matrix. Let $\left(\pi_{\phi^{\omega}}, H_{\phi^{\omega}}, \xi_{\phi^{\omega}}\right)$ be the GNS-triple of $\phi^{\omega}$. The above theorem, in particular, says that the von Neumann algebra $M=\pi_{\phi \omega}\left(\mathcal{O}_{A}\right)^{\prime \prime}$ becomes a factor.

2.3. AF-algebras. The following results are based on [SV] Theorem I.3.12]. Consider an AF-algebra $B=\overline{\bigcup_{n>0} B_{n}}$, where $\left\{B_{n}\right\}_{n=0}^{\infty}$ is an increasing family of finite $C^{*}$-subalgebras. We assume that $B_{0}=\mathbb{C} 1$. We define a maximal abelian subalgebra $C$ of $B$ as follows. Let $C_{0}=B_{0}$ and let $C_{n+1}$ be the $C^{*}$-subalgebra generated by $C_{n}$ and $D_{n+1}$, where $D_{n+1}$ is a masa of $B_{n+1}$, containing $C_{n}$. We define $C=\overline{\bigcup_{n>0} C_{n}}$. One can check that $C$ is a masa of $B$. There is a conditional expectation $\Psi$ from $B$ onto $C$, and there is a topological dynamical system $(\Omega, \Gamma)$ such that $C \simeq C(\Omega)$, $B=\overline{\operatorname{span}}\{f u \mid f \in C(\Omega), u \in \Gamma\}$ and $\Gamma=\bigcup_{n \geq 0} \Gamma_{n}$, where $\Gamma_{n}$ consists of all unitaries $u$ in $B_{n}$ with $u C_{n} u^{*}=C_{n}$. Let $\nu$ be a $\Gamma$-quasi-invariant probability measure on $\Omega$. It induces a state $\psi=\nu \circ \Psi$ of $B$. Let $\left(\pi_{\psi}, H_{\psi}, \xi_{\psi}\right)$ be the GNS-triple of $\psi$. Then we obtain the following:

(1) $\pi_{\psi}(C)^{\prime \prime}$ is a masa in $\pi_{\psi}(B)^{\prime \prime}$.

(2) $\pi_{\psi}(C)^{\prime \prime} \simeq L^{\infty}(\Omega, \nu)$.

(3) The conditional expectation $\Psi$ can extend to $\pi_{\psi}(B)^{\prime \prime}$ whose image is $\pi_{\psi}(C)^{\prime \prime}$.

\section{LemMata}

We denote by $\mathcal{O}_{A}^{\alpha^{\omega}}$ the fixed-point algebra under $\alpha^{\omega}$. We first introduce an equivalence relation on the index set $I=\{1, \ldots, N\}$. We say that $i$ is equivalent to $j$ if there are $\xi, \eta \in \mathcal{W}_{A}$ such that $s(\xi)=i, s(\eta)=j, r(\xi)=r(\eta)$ and $\omega_{\xi}=\omega_{\eta}$. It is easy to check that this is an equivalence relation. We obtain the corresponding disjoint union $I=I_{1}^{\omega} \cup \cdots \cup I_{n_{\omega}}^{\omega}$. Note that if $\alpha^{\omega}$ is the gauge action, then this decomposition coincides with the one with respect to the period of $A$. Set $P_{I_{k}^{\omega}}=$ $\sum_{i \in I_{k}^{\omega}} P_{i}$. Our goal in this section is to prove the following lemma.

\section{Lemma 3.1.}

$$
Z\left(\pi_{\phi^{\omega}}\left(\mathcal{O}_{A}^{\alpha^{\omega}}\right)^{\prime \prime}\right)=\pi_{\phi^{\omega}}\left(\mathcal{O}_{A}^{\alpha^{\omega}}\right)^{\prime \prime} \cap \pi_{\phi^{\omega}}\left(\mathcal{O}_{A}^{\alpha^{\omega}}\right)^{\prime}=\bigoplus_{k=1}^{n_{\omega}} \mathbb{C} \pi_{\phi^{\omega}}\left(P_{I_{k}^{\omega}}\right)
$$


We need some lemmata to show the above.

Lemma 3.2. The fixed-point algebra $\mathcal{O}_{A}^{\alpha^{\omega}}$ is an AF-algebra.

Proof. Set $F_{t}^{i}=\operatorname{span}\left\{S_{\xi} P_{i} S_{\eta}^{*} \mid \omega_{\xi}=\omega_{\eta}=t\right\}$ for $t \in\left\{\omega_{\xi} \mid \xi \in \mathcal{W}_{A}\right\}$. Since $\left\{S_{\xi} P_{i} S_{\eta}^{*}\right\}$ gives the matrix units, $F_{t}^{i}$ is a simple finite-dimensional $C^{*}$-algebra. We can define finite-dimensional $C^{*}$-algebras $F_{n}$ as follows:

$$
\begin{aligned}
F_{-1} & =\mathbb{C} 1 \\
F_{n} & =\bigvee_{i \in I} \bigvee_{t \in K_{n}^{i}} F_{t}^{i}=\bigoplus_{i \in I} \bigoplus_{t \in K_{n}^{i}} F_{t}^{i} \text { for } n \geq 0,
\end{aligned}
$$

where $\omega_{\min }=\min \left\{\omega_{i} \mid i \in I\right\}$ and

$$
K_{n}^{i}=\left\{\omega_{\xi} \mid \xi \in \mathcal{W}_{A}, A(r(\xi), i)=1, n \omega_{\min }-\omega_{i}<\omega_{\xi} \leq n \omega_{\min }\right\} .
$$

Indeed, let $S_{\xi_{1}} P_{i} S_{\eta_{1}}^{*} \in F_{t}^{i}$ for $t \in K_{n}^{i}$ and $S_{\xi_{2}} P_{j} S_{\eta_{2}}^{*} \in F_{s}^{j}$ for $s \in K_{n}^{j}$. We assume that $S_{\xi_{1}} P_{i} S_{\eta_{1}}^{*} S_{\xi_{2}} P_{j} S_{\eta_{2}}^{*} \neq 0$. If $\left|\eta_{1}\right|=\left|\xi_{2}\right|$, then $\eta_{1}=\xi_{2}$ and thus $s=t$ and $i=j$. We now suppose that $\left|\eta_{1}\right| \neq\left|\xi_{2}\right|$. Without loss of generality, we may assume that $\left|\eta_{1}\right|<\left|\xi_{2}\right|$. Since $P_{i} S_{\eta_{1}}^{*} S_{\xi_{2}} P_{j} \neq 0$, we have $P_{i} S_{\eta_{1}}^{*} S_{\xi_{2}} P_{j}=S_{\xi} P_{j}$ for some $\xi$ with $\xi_{2}=\eta_{1} \cdot \xi$ and $s(\xi)=i$. Hence we obtain $\omega_{i} \leq \omega_{\xi}$. However,

$$
\omega_{\xi_{2}}=\omega_{\eta_{1}}+\omega_{\xi}>n \omega_{\min }-\omega_{i}+\omega_{i}=n \omega_{\min } .
$$

Thus $n \omega_{\min }<\omega_{\xi_{2}} \leq n \omega_{\min }$ and this is a contradiction.

We next show that $F_{n}$ is a $C^{*}$-subalgebra of $F_{n+1}$. Let $S_{\xi} P_{i} S_{\eta}^{*} \in F_{t}^{i}$ with $t \in K_{n}^{i}$. If $(n+1) \omega_{\min }-\omega_{i}<t$, then we have $S_{\xi} P_{i} S_{\eta}^{*} \in F_{n+1}$. If $t \leq(n+1) \omega_{\min }-\omega_{i}$, then we have

$$
S_{\xi} P_{i} S_{\eta}^{*}=\sum_{j \in I} A(i, j) S_{\xi} S_{i} P_{j} S_{i}^{*} S_{\eta}^{*} \in F_{n+1} .
$$

We can define an AF-algebra $F=\overline{\bigcup_{n} F_{n}}$.

We claim that $F=\mathcal{O}_{A}^{\alpha^{\omega}}$. It is clear that $F \subseteq \mathcal{O}_{A}^{\alpha}{ }^{\omega}$. To show the converse, we need the conditional expectation. If $\omega_{i} / \omega_{j} \in \mathbb{Q}$ for all $i, j \in I$, then we can define the faithful conditional expectation from $\mathcal{O}_{A}$ onto $\mathcal{O}_{A}^{\alpha^{\omega}}$ by the integration on $\mathbb{T}$. If not, we consider an action $\tilde{\alpha}$ of $\mathbb{T}^{N}$ such that $\tilde{\alpha}_{z}\left(S_{i}\right)=z_{i} S_{i}$ for $z \in\left(z_{1}, \ldots, z_{N}\right) \in \mathbb{T}^{N}$. Since there is the embedding of $\mathbb{R}$ into $\mathbb{T}^{N}, t \mapsto\left(e^{\sqrt{-1} \omega_{1} t}, \ldots, e^{\sqrt{-1} \omega_{N} t}\right)$, we can consider the closure of $\mathbb{R}$ in $\mathbb{T}^{N}$ via this embedding. Therefore the conditional expectation is given by the integration on the compact group $\overline{\mathbb{R}}$. One can easily check that the fixed-point algebra under $\left.\tilde{\alpha}\right|_{\mathbb{R}}$ coincides with $\mathcal{O}_{A}^{\alpha^{\omega}}$ and thus we can show that $\mathcal{O}_{A}^{\alpha^{\omega}}=F$ by using this conditional expectation.

We will need one more lemma. Let $p$ be the period of the matrix $A$. We define partial isometries for $m \in \mathbb{N}, i \in I$ by

$$
\theta_{m}^{(i)}=\sum_{\xi, \eta \in L_{i}(m p)} S_{\xi} S_{\eta} P_{i} S_{\xi}^{*} S_{\eta}^{*},
$$

where $L_{i}(n)=\left\{\xi \in \mathcal{W}_{A}|s(\xi)=i, A(r(\xi), i)=1,| \xi \mid=n\right\}$ is the set of all loops of $i$ with length $n$. Note that $\theta_{m}^{(i)}$ is self-adjoint. We define the tracial state by $\psi^{\omega}=\left.\phi^{\omega}\right|_{\mathcal{O}_{A}^{\alpha}}$ on $\mathcal{O}_{A}^{\alpha^{\omega}}$, and use the same symbol $\psi^{\omega}$ for its normal extension to $\pi_{\psi^{\omega}}\left(\mathcal{O}_{A}^{\alpha^{\omega}}\right)^{\prime \prime}$ for simplicity. 
Lemma 3.3. Let $f \in \pi_{\psi^{\omega}}\left(C\left(\Omega_{A}\right)\right)^{\prime \prime}$ and $a \in \pi_{\psi^{\omega}}\left(\mathcal{O}_{A}^{\alpha^{\omega}}\right)^{\prime \prime}$. Then for any $i \in I$,

$$
\lim _{m \rightarrow \infty} \psi^{\omega}\left(\theta_{m}^{(i)} f \theta_{m}^{(i)} a\right)=\psi^{\omega}\left(P_{i} f\right) \psi^{\omega}\left(P_{i} a\right) x_{i} y_{i}^{2},
$$

where $y=\left(y_{1}, \ldots, y_{N}\right)$ is the left Perron eigenvector of $A_{\omega}$ with $\sum_{i \in I} x_{i} y_{i}=p$.

Proof. Note that $C\left(\Omega_{A}\right) \simeq \overline{\operatorname{span}}\left\{S_{\xi} S_{\xi}^{*} \mid \xi \in \mathcal{W}_{A}\right\}$ is a masa in the AF-algebra $\mathcal{O}_{A}^{\alpha^{\omega}}$. We denote by $\Psi$ the conditional expectation from $\pi_{\psi^{\omega}}\left(\mathcal{O}_{A}^{\alpha^{\omega}}\right)^{\prime \prime}$ onto $\pi_{\psi^{\omega}}\left(C\left(\Omega_{A}\right)\right)^{\prime \prime} \simeq$ $L^{\infty}\left(\Omega_{A}, \nu\right)$. We first prove the lemma for $f \in C\left(\Omega_{A}\right)$ and $a \in \mathcal{O}_{A}^{\alpha^{\omega}}$. Remark that $\psi^{\omega}=\nu \circ \Psi$. We may assume that $a \in C\left(\Omega_{A}\right)$. Indeed, if the statement holds for $\Psi(a)$ instead of $a$, then since $\theta_{m}^{(i)} f \theta_{m}^{(i)} \in C\left(\Omega_{A}\right)$, we will have

$$
\begin{aligned}
\lim _{m \rightarrow \infty} \psi^{\omega}\left(\theta_{m}^{(i)} f \theta_{m}^{(i)} a\right) & =\lim _{m \rightarrow \infty} \psi^{\omega}\left(\theta_{m}^{(i)} f \theta_{m}^{(i)} \Psi(a)\right) \\
& =\psi^{\omega}\left(P_{i} f\right) \psi^{\omega}\left(P_{i} \Psi(a)\right) x_{i} y_{i}^{2} \\
& =\psi^{\omega}\left(P_{i} f\right) \psi^{\omega}\left(P_{i} a\right) x_{i} y_{i}^{2} .
\end{aligned}
$$

It suffices to check the statement for $f=S_{\zeta_{1}} P_{j_{1}} S_{\zeta_{1}}^{*}, a=S_{\zeta_{2}} P_{j_{2}} S_{\zeta_{2}}^{*}$ with $\left|\zeta_{1}\right|=$ $k p,\left|\zeta_{2}\right|=l p$ and $s\left(\zeta_{1}\right)=s\left(\zeta_{2}\right)=i$. In this case, for sufficiently large $m$ we have

$$
\theta_{m}^{(i)} f \theta_{m}^{(i)} a=\sum_{\xi^{\prime}, \eta^{\prime}} S_{\zeta_{2}} S_{\xi^{\prime}} S_{\zeta_{1}} S_{\eta^{\prime}} P_{i} S_{\eta^{\prime}}^{*} S_{\zeta_{1}}^{*} S_{\xi^{\prime}}^{*} S_{\zeta_{2}}^{*}
$$

where $\xi^{\prime}$ and $\eta^{\prime}$ run over all admissible words from $j_{2}, j_{1}$ to $i$ with $\left|\xi^{\prime}\right|=(m-l) p$ and $\left|\eta^{\prime}\right|=(m-k) p$. Therefore

$$
\begin{aligned}
\psi^{\omega}\left(\theta_{m}^{(i)} f \theta_{m}^{(i)} a\right) & =e^{-\beta \omega_{\zeta_{2}}} A_{\omega}^{(m-l) p}\left(j_{2}, i\right) e^{-\beta \omega_{\zeta_{1}}} A_{\omega}^{(m-k) p}\left(j_{1}, i\right) x_{i} \\
& \longrightarrow e^{-\beta \omega_{\zeta_{2}}} x_{j_{2}} y_{i} e^{-\beta \omega_{\zeta_{1}}} x_{j_{1}} y_{i} x_{i} \quad(m \rightarrow \infty) \\
& =\psi^{\omega}(f) \psi^{\omega}(a) x_{i} y_{i}^{2} .
\end{aligned}
$$

Next let $f \in L^{\infty}\left(\Omega_{A}, \nu\right)$. We choose $g \in C\left(\Omega_{A}\right)$ with $\left\|(f-g) \xi_{\psi^{\omega}}\right\|<\varepsilon$. Then

$$
\begin{aligned}
\left|\psi^{\omega}\left(\theta_{m}^{(i)} f \theta_{m}^{(i)} a\right)-\psi^{\omega}\left(P_{i} f\right) \psi^{\omega}\left(P_{i} a\right) x_{i} y_{i}^{2}\right| & \leq\left|\psi^{\omega}\left(\theta_{m}^{(i)}(f-g) \theta_{m}^{(i)} a\right)\right| \\
& +\left|\psi^{\omega}\left(\theta_{m}^{(i)} g \theta_{m}^{(i)} a\right)-\psi^{\omega}\left(P_{i} g\right) \psi^{\omega}\left(P_{i} a\right) x_{i} y_{i}^{2}\right| \\
& +\left|\psi^{\omega}\left(P_{i}(f-g)\right) \psi^{\omega}\left(P_{i} a\right) x_{i} y_{i}^{2}\right|,
\end{aligned}
$$

and we get the following estimate of the first term:

$$
\begin{aligned}
\left|\psi^{\omega}\left(\theta_{m}^{(i)}(f-g) \theta_{m}^{(i)} a\right)\right| & =\left|\psi^{\omega}\left(\theta_{m}^{(i)} a \theta_{m}^{(i)}(f-g)\right)\right| \\
& \leq \psi^{\omega}\left(\theta_{m}^{(i)} a \theta_{m}^{(i)} \theta_{m}^{(i)} a^{*} \theta_{m}^{(i)}\right)^{1 / 2} \psi^{\omega}\left((f-g)^{*}(f-g)\right)^{1 / 2} \\
& \leq\|a\|\left\|\mid(f-g) \xi_{\psi^{\omega}}\right\|,
\end{aligned}
$$

because $\psi^{\omega}$ is tracial. In a similar way, we can show the statement for $a \in$ $\pi_{\psi^{\omega}}\left(\mathcal{O}_{A}^{\alpha^{\omega}}\right)^{\prime \prime}$.

We will use the following folklore among specialists (e.g. see [Izu]).

Lemma 3.4 (【zu, Lemma 4.1]). Let $B$ be a unital $C^{*}$-algebra, $\phi$ a state of $B$ and $\left(\pi_{\phi}, H_{\phi}, \xi_{\phi}\right)$ the GNS-triple of $\phi$. We assume that the cyclic vector $\xi_{\phi}$ is a separating vector for $\pi_{\phi}(B)^{\prime \prime}$. Let $C$ be a unital $C^{*}$-subalgebra of $B$ and $\psi$ the restriction of $\phi$ to $C$. Then $\left(\left.\pi_{\phi}\right|_{C}, H_{\phi}\right)$ is quasi-equivalent to the GNS-representation $\left(\pi_{\psi}, H_{\psi}\right)$ of $\psi$.

Now we have the necessary ingredients for the proof of Lemma 3.1. 
Proof of Lemma 3.1. It is easy to show that $\pi_{\phi^{\omega}}\left(P_{I_{k}^{\omega}}\right) \in Z\left(\pi_{\phi^{\omega}}\left(\mathcal{O}_{A}^{\alpha^{\omega}}\right)^{\prime \prime}\right)$ for $k=$ $1, \ldots, n_{\omega}$. By Lemma 3.4, $\pi_{\phi^{\omega}}\left(\mathcal{O}_{A}^{\alpha^{\omega}}\right)^{\prime \prime}$ is isomorphic to $\pi_{\psi^{\omega}}\left(\mathcal{O}_{A}^{\alpha^{\omega}}\right)^{\prime \prime}$. It therefore suffices to show that $Z\left(\pi_{\psi^{\omega}}\left(\mathcal{O}_{A}^{\alpha^{\omega}}\right)^{\prime \prime}\right)=\bigoplus_{k=1}^{n_{\omega}} \mathbb{C} P_{I_{k}^{\omega}}$. Let $z \in Z\left(\pi_{\psi^{\omega}}\left(\mathcal{O}_{A}^{\alpha^{\omega}}\right)^{\prime \prime}\right)$ be a non-trivial projection. Since $L^{\infty}\left(\Omega_{A}\right)$ is a masa in $\pi_{\psi^{\omega}}\left(\mathcal{O}_{A}^{\alpha^{\omega}}\right)^{\prime \prime}$, we have $z \in$ $L^{\infty}\left(\Omega_{A}\right)$. We can apply Lemma 3.3 to $f=P_{i} z$ :

$$
\lim _{m \rightarrow \infty} \psi^{\omega}\left(\theta_{m}^{(i)} z \theta_{m}^{(i)} a\right)=\psi^{\omega}\left(P_{i} z\right) \psi^{\omega}\left(P_{i} a\right) x_{i} y_{i}^{2} .
$$

On the other hand, since $z$ is centered, we get

$$
\lim _{m \rightarrow \infty} \psi^{\omega}\left(\theta_{m}^{(i)} z \theta_{m}^{(i)} a\right)=\lim _{m \rightarrow \infty} \psi^{\omega}\left(\theta_{m}^{(i)} \theta_{m}^{(i)} z a\right)=\psi^{\omega}\left(P_{i}\right) \psi^{\omega}\left(P_{i} z a\right) x_{i} y_{i}^{2} .
$$

Therefore

$$
\psi^{\omega}\left(P_{i}\right) \psi^{\omega}\left(P_{i} z a\right)=\psi^{\omega}\left(P_{i} z\right) \psi^{\omega}\left(P_{i} a\right) .
$$

Since $\psi^{\omega}$ is faithful on $\pi_{\psi^{\omega}}\left(\mathcal{O}_{A}^{\alpha^{\omega}}\right)^{\prime \prime}$ and $a$ is arbitrary, we get

$$
P_{i} z=\frac{\psi^{\omega}\left(P_{i} z\right)}{\psi^{\omega}\left(P_{i}\right)} P_{i} .
$$

Therefore we obtain $z=\sum_{i \in I} c_{i} P_{i}$ for $c_{i} \in\{0,1\}$. If $c_{i} \neq 0$ for $i \in I_{k}^{\omega}$, then there are admissible words $\xi, \eta$ with $s(\xi)=i, s(\eta)=j, r(\xi)=r(\eta)$ and $\omega_{\xi}=\omega_{\eta}$ for $j \in I_{k}^{\omega}$, and $z$ must commute with $S_{\xi} S_{\eta}^{*} \in \mathcal{O}_{A}^{\alpha^{\omega}}$. Hence we have $z=\sum_{k=1}^{n_{\omega}} c_{k} P_{I_{k}^{\omega}}$ for $c_{k} \in\{0,1\}$.

\section{MAIN THEOREM}

We first review some notations in Con. Let $(M, \mathbb{R}, \sigma)$ be a $W^{*}$-dynamical system. For $f \in L^{1}(\mathbb{R})$, we define a $\sigma$-weakly continuous linear map on $M$ by

$$
\sigma_{f}(x)=\int f(t) \sigma_{t}(x) d t \quad \text { for } x \in M
$$

The Arveson spectrum of $\sigma$ is defined by

$$
\operatorname{Sp}(\sigma)=\bigcap\left\{Z(f) \mid f \in L^{1}(\mathbb{R}), \sigma_{f}=0\right\},
$$

where $Z(f)=\{r \in \hat{\mathbb{R}} \mid \hat{f}(r)=0\}$ and $\hat{\mathbb{R}}$ is the dual group of $\mathbb{R}$. Then the Connes spectrum of $\sigma$ is defined by

$$
\Gamma(\sigma)=\bigcap_{p} \operatorname{Sp}\left(\left.\sigma\right|_{p M p}\right)
$$

where $p$ runs over all non-zero projections in $Z\left(M^{\sigma}\right)=M^{\sigma} \cap\left(M^{\sigma}\right)^{\prime}$. Note that $\Gamma(\sigma) \subseteq \operatorname{Sp}\left(\left.\sigma\right|_{p M p}\right)$ holds for any non-zero projection $p$ in $M^{\sigma}$.

For each $i \in I$, let $G_{i}$ be the closed additive subgroup of $\mathbb{R}$ generated by $\beta \omega_{\xi}$ for all loops $\xi$ with $s(\xi)=i$ and $G$ the closed additive subgroup generated by $\beta \omega_{\xi}$ for all circles $\xi$.

Lemma 4.1. For any $i \in I, G=G_{i}$.

Proof. It is clear that $G \subseteq G_{i}$. Conversely, let $\xi$ be a loop with $s(\xi)=i$. Then there are circles $\xi(1), \ldots, \xi(n)$ such that $\omega_{\xi}=\omega_{\xi(1)}+\cdots+\omega_{\xi(n)}$. Thus $G_{i} \subseteq G$.

We will prove the following main theorem.

Theorem 4.2. (1) If $\omega_{\xi} / \omega_{\eta} \in \mathbb{Q}$ for all circles $\xi, \eta$, then $M=\pi_{\psi^{\omega}}\left(\mathcal{O}_{A}\right)^{\prime \prime}$ is the AFD type $\mathrm{III}_{\lambda}$ factor for $\lambda=e^{-r}$, where $G=r \mathbb{Z}$ for some $r \in \mathbb{R}_{+}$. 
(2) If $\omega_{\xi} / \omega_{\eta} \notin \mathbb{Q}$ for some circles $\xi, \eta$, then $M=\pi_{\psi^{\omega}}\left(\mathcal{O}_{A}\right)^{\prime \prime}$ is the AFD type III 1 factor.

Proof. Since $\phi^{\omega}$ is $\alpha^{\omega}$-invariant, $\alpha^{\omega}$ can be extended to an action on $M$. We use the same symbol $\phi^{\omega}$ for its normal extension. Let $\sigma^{\phi^{\omega}}$ be the modular automorphism group for $\phi^{\omega}$, which satisfies $\sigma_{t}^{\phi^{\omega}}=\alpha_{-\beta t}^{\omega}$ for $t \in \mathbb{R}$. We first claim that $M^{\sigma}=$ $\pi_{\phi^{\omega}}\left(\mathcal{O}_{A}^{\sigma}\right)^{\prime \prime}$. One can check that the conditional expectation from $\mathcal{O}_{A}$ onto $\mathcal{O}_{A}^{\alpha^{\omega}}$ in the proof of Lemma 3.2 can extend to the one on $\pi_{\phi^{\omega}}\left(\mathcal{O}_{A}\right)^{\prime \prime}$. Thus by the approximation arguments, we can obtain our claim.

By Lemma 3.1, we obtain $\Gamma\left(\sigma^{\phi^{\omega}}\right)=\bigcap_{k} \operatorname{Sp}\left(\left.\sigma^{\phi^{\omega}}\right|_{P_{I_{k}^{\omega}}^{\omega} M P_{I_{k} \omega}}\right)$. Since $\operatorname{Sp}\left(\left.\sigma^{\phi^{\omega}}\right|_{P_{i} M P_{i}}\right)$ $\subseteq \operatorname{Sp}\left(\left.\sigma^{\phi^{\omega}}\right|_{P_{I_{k}^{\omega}} M P_{I_{k} \omega}}\right)$ for $i \in I_{k}^{\omega}$, we have $\Gamma\left(\sigma^{\phi^{\omega}}\right)=\bigcap_{i \in I} \operatorname{Sp}\left(\left.\sigma^{\phi^{\omega}}\right|_{P_{i} M P_{i}}\right)$.

We now claim that $\operatorname{Sp}\left(\left.\sigma^{\phi^{\omega}}\right|_{P_{i} M P_{i}}\right)=G_{i}$ for each $i \in I$. Let $\xi, \eta$ be loops with $s(\xi)=s(\eta)=i$. If $\left.f \in \operatorname{Ker} \sigma^{\phi_{\omega}}\right|_{P_{i} M P_{i}}$, then

$$
0=\sigma_{f}^{\phi_{\omega}}\left(P_{i} S_{\xi} S_{\eta}^{*} P_{i}\right)=\hat{f}\left(\beta\left(\omega_{\xi}-\omega_{\eta}\right)\right) P_{i} S_{\xi} S_{\eta}^{*} P_{i} .
$$

Since $P_{i} S_{\xi} S_{\eta}^{*} P_{i} \neq 0$, we have $\beta\left(\omega_{\xi}-\omega_{\eta}\right) \in \operatorname{Sp}\left(\left.\sigma^{\phi^{\omega}}\right|_{P_{i} M P_{i}}\right)$. Thus a group generated by $\beta \omega_{\xi}$ for all loops $\xi$ with $s(\xi)=i$ is contained in $\operatorname{Sp}\left(\left.\sigma^{\phi^{\omega}}\right|_{P_{i} M P_{i}}\right)$. Since $\operatorname{Sp}\left(\left.\sigma^{\phi^{\omega}}\right|_{P_{i} M P_{i}}\right)$ is closed, $G_{i} \subseteq \operatorname{Sp}\left(\left.\sigma^{\phi^{\omega}}\right|_{P_{i} M P_{i}}\right)$ holds. Conversely let $r \in \mathbb{R} \backslash G_{i}$. Choose a function $f \in L^{1}(\mathbb{R})$ with $\hat{f}(r) \neq 0$ and $\left.\hat{f}\right|_{G_{i}}=0$. We have

$$
\left.\sigma_{f}^{\phi_{\omega}}\right|_{P_{i} M P_{i}}\left(P_{i} S_{\xi} S_{\eta}^{*} P_{i}\right)=\hat{f}\left(\beta\left(\omega_{\xi}-\omega_{\eta}\right)\right) P_{i} S_{\xi} S_{\eta}^{*} P_{i} .
$$

If $P_{i} S_{\xi} S_{\eta}^{*} P_{i} \neq 0$, then we have $s(\xi)=s(\eta)=i$ and $A(r(\xi), j)=A(r(\eta), j)=1$ for some $j \in I$. Since $A$ is irreducible, there is an admissible word $\zeta$ with $s(\zeta)=j$ and $A(r(\zeta), i)=1$. Two admissible words $\xi \cdot \zeta, \eta \cdot \zeta$ are loops with $s(\xi \cdot \zeta)=s(\eta \cdot \zeta)=i$. Hence

$$
\beta\left(\omega_{\xi}-\omega_{\eta}\right)=\beta\left(\omega_{\xi}+\omega_{\zeta}-\omega_{\eta}-\omega_{\zeta}\right)=\beta\left(\omega_{\xi \cdot \zeta}-\omega_{\eta \cdot \zeta}\right) \in G_{i}
$$

We therefore obtain $\left.f \in \operatorname{Ker} \sigma^{\phi_{\omega}}\right|_{P_{i} M P_{i}}$. It follows from Lemma 4.1 that $\Gamma\left(\sigma^{\phi^{\omega}}\right)=$ $G$. In the case (1), we have $G=r \mathbb{Z}$ for some $r \in \mathbb{R}_{+}$and $\lambda$ is determined by $e^{-r}$.

Example 4.3. Let $\mathbb{F}_{n}$ be the free group with the canonical generators $a_{1}, \ldots, a_{n}$ and $S=\left\{a_{1}, a_{1}^{-1}, \ldots, a_{n}, a_{n}^{-1}\right\}$ the generating set. The corresponding Cayley graph is the homogeneous tree with degree $2 n$. We define a compact space by

$$
\Omega=\left\{\left(x_{i}\right)_{i=1}^{\infty} \mid x_{i} \neq x_{i+1}^{-1}\right\} \subseteq \prod_{i=1}^{\infty} S .
$$

Note that $\Omega$ is compact and $\Gamma$ acts on $\Omega$ by left multiplications. We remark that $\Omega$ coincides with the hyperbolic boundary $\partial \mathbb{F}_{n}$ of $\mathbb{F}_{n}$. In Spi], Spielberg showed the identification $\mathcal{O}_{A} \simeq C(\Omega) \rtimes \mathbb{F}_{n}$, where

$$
A=\left(\begin{array}{ccccccc}
1 & 0 & 1 & 1 & \cdots & 1 & 1 \\
0 & 1 & 1 & 1 & \cdots & 1 & 1 \\
1 & 1 & 1 & 0 & \cdots & 1 & 1 \\
1 & 1 & 0 & 1 & \cdots & 1 & 1 \\
\vdots & \vdots & \vdots & \vdots & \ddots & \vdots & \vdots \\
1 & 1 & 1 & 1 & \cdots & 1 & 0 \\
1 & 1 & 1 & 1 & \cdots & 0 & 1
\end{array}\right)(2 n \times 2 n \text {-matrix })
$$


We now apply Theorem 4.2 to $\mathcal{O}_{A} \simeq C(\Omega) \rtimes \mathbb{F}_{n}$. Note that the canonical masa $C\left(\Omega_{A}\right)$ of $\mathcal{O}_{A}$ coincides with $C(\Omega)$. Let $\omega=\left(\omega_{x}\right)_{x \in S} \in \mathbb{R}_{+}^{2 n}$ and let $\nu$ be the corresponding probability measure on $\Omega$, which induces the KMS state for $\alpha^{\omega}$. By Theorem 4.2, we have the following:

(1) If $\omega_{x} / \omega_{y} \in \mathbb{Q}$ for all $x, y \in S$, then $L^{\infty}(\Omega, \nu) \rtimes \mathbb{F}_{n}$ is the AFD type $\operatorname{III}_{\lambda}$ factor for some $0<\lambda<1$.

(2) If $\omega_{x} / \omega_{y} \notin \mathbb{Q}$ for some $x, y \in S$, then $L^{\infty}(\Omega, \nu) \rtimes \mathbb{F}_{n}$ is the AFD type $\mathrm{III}_{1}$ factor.

Let $\mu$ be a probability measure on $\mathbb{F}_{n}$ with $\operatorname{supp} \mu=S$. By Oka], the random walk with law $\mu$ induces the harmonic measure $\nu$ on $\Omega$ such that the modular automorphism group of the state $\nu \circ \Phi$ has the form $\alpha^{\omega}$ for some $\omega=\left(\omega_{x}\right)_{x \in S} \in \mathbb{R}_{+}^{2 n}$. Therefore the above result also means that we determine the type of harmonic measures on $\Omega$ (cf. $\underline{\mathrm{RR}}$ ).

Remark 4.4. We can also prove the same results for $\mathcal{O}_{\Gamma}$ in Oka in the same way, where $\Gamma$ is an amalgamated free product group ${ }_{H} G_{i}$. Here we will give a sketch of the proof.

Let $I$ be a finite index set and $G_{i}$ a group containing a copy of a group $H$ as a subgroup for $i \in I$. We assume that $G_{i}$ is finite for simplicity. $\mathcal{O}_{\Gamma}$ is the universal $C^{*}$-algebra generated by partial isometries $S_{g}, g \in \bigcup_{i \in I} G_{i} \backslash H$ and unitaries $U_{h}$, $h \in H$ satisfying certain conditions (see [Oka]). We use some symbols in [Oka]. For $\omega=\left(\omega_{i}\right)_{i \in I} \in \mathbb{R}_{+}^{|I|}$, we consider the action $\alpha^{\omega}$ of $\mathbb{R}$ given by

$$
\begin{aligned}
& \alpha_{t}^{\omega}\left(S_{g}\right)=e^{\sqrt{-1} \omega_{i} t} S_{g} \quad \text { for } \quad i \in I, g \in G_{i} \backslash H, \\
& \alpha_{t}^{\omega}\left(U_{h}\right)=U_{h} \quad \text { for } \quad h \in H \text {, }
\end{aligned}
$$

where $|I|$ is the cardinality of $I$. Remark that there is an identification $\mathcal{O}_{\Gamma} \simeq$ $C(\Omega) \rtimes \Gamma$ for some compact space $\Omega$ ( $0 \mathrm{ka}$ Theorem 5.3]). Let $\Phi$ be the canonical conditional expectation from $C(\Omega) \rtimes \Gamma$ onto $C(\Omega)$. It was shown that there is the unique $\beta$-KMS state $\phi=\nu \circ \Phi$ for $\alpha^{\omega}$, where $\nu$ is the corresponding probability measure on $\Omega$. However the difference from the above example is that $C(\Omega)$ may not be a masa of the fixed-point algebra under $\alpha^{\omega}$. Therefore we need some arguments to obtain the similar result for $\mathcal{O}_{\Gamma}$. Choose a masa $C$ containing $C(\Omega)$. We assume that $\Gamma=*_{H} G_{i}$ satisfies the following condition:

For any $i \in I$, there is an element $\gamma_{i}=g_{1} \cdots g_{n} \in \Gamma$ such that $h \gamma_{i} H \neq \gamma_{i} H$ for any $(e \neq) h \in H$, where $g_{k} \in G_{i_{k}} \backslash H$ with $i=i_{1} \neq i_{2}, i_{2} \neq i_{3}, \ldots, i_{n-1} \neq i_{n}$.

We remark that the above assumption holds if $\Gamma=*_{H} G_{i}$ satisfies the condition of [Oka, Corollary 6.4]. Fix $\gamma_{i}$ satisfying the above. Let $\psi$ be the restriction of $\phi$ on the fixed-point algebra under $\mathcal{O}_{\Gamma}^{\alpha^{\omega}}$. For $g \in G_{i} \backslash H$, we set

$$
\theta_{m}^{(g)}=\sum_{\xi, \eta} S_{\xi} S_{\gamma_{i}} S_{\eta} P_{g} S_{\xi}^{*} S_{\gamma_{i}}^{*} S_{\eta}^{*}
$$

where $\xi, \eta$ run over all words from $g$ to an element, which is not in $G_{i}$, with length $m$ if $|I|>2$ and length $2 m$ if $|I|=2$. Let $\pi_{\psi}$ be the GNS-representation of $\psi$. Then we will get the similar result of Lemma 3.3.

Lemma 4.5. For $f \in \pi_{\psi}(C)^{\prime \prime}$ and $a \in \pi_{\psi}\left(\mathcal{O}_{\Gamma}^{\alpha^{\omega}}\right)^{\prime \prime}$, we have

$$
\lim _{m \rightarrow \infty} \psi\left(\theta_{m}^{(g)} f \theta_{m}^{(g)} a\right)=\psi\left(P_{g} f\right) \psi\left(P_{g} a\right) x_{g} y_{g}^{2} z_{\gamma_{i}}
$$

where $x_{g}, y_{g}, z_{\gamma_{i}}$ are some constants. 
Using this lemma, we can similarly prove the following

\section{Proposition 4.6.}

$$
Z\left(\pi_{\psi}\left(\mathcal{O}_{\Gamma}^{\alpha^{\omega}}\right)^{\prime \prime}\right)=\left\{\begin{array}{cll}
\bigoplus_{i \in I} \mathbb{C} P_{i} & \text { if } & |I|=2 \\
\mathbb{C} 1 & \text { if } & |I|>2 .
\end{array}\right.
$$

Hence we can compute the Connes spectrum of the modular automorphism group in the similar way. This gives a generalization on $\mathrm{RR}$.

Corollary 4.7. Let $\mathcal{O}_{\Gamma}, \omega, \phi, \nu$ be the above and $\pi_{\phi}$ the GNS-representation of $\phi$. Then

(1) If $\omega_{i} / \omega_{j} \in \mathbb{Q}$ for any $i, j \in I$, then $\pi_{\phi}\left(\mathcal{O}_{\Gamma}\right)^{\prime \prime} \simeq L^{\infty}(\Omega, \nu) \rtimes \Gamma$ is the AFD type $\mathrm{III}_{\lambda}$ factor for some $0<\lambda<1$.

(2) If $\omega_{i} / \omega_{j} \notin \mathbb{Q}$ for some $i, j \in I$, then $\pi_{\phi}\left(\mathcal{O}_{\Gamma}\right)^{\prime \prime} \simeq L^{\infty}(\Omega, \nu) \rtimes \Gamma$ is the AFD type $\mathrm{III}_{1}$ factor.

\section{ACKNOWLEDGMENT}

The author expresses his gratitude to Masaki Izumi and Yoshimichi Ueda for various comments and important suggestions. He is grateful to Hiroki Matui and Takeshi Katsura for useful discussions. He also thanks the referee for careful reading. The present work was done while the author stayed at the Mathematical Sciences Research Institute from the summer of 2000 to the spring of 2001 . He thanks them for their hospitality.

\section{REFERENCES}

[Con] A. Connes, Une classification des facteurs de type III, (French) Ann. Sci. Ecole Norm. Sup. 6 (1973), 133-252. MR 49:5865

[Cun] J. Cuntz, Simple $C^{*}$-algebras generated by isometries, Commun. Math. Phys. 57 (1977), 173-185. MR 57:7189]

[CK] J. Cuntz and W. Krieger, A class of $C^{*}$-algebras and topological Markov chains, Invent. Math. 56 (1980) 251-268. MR 82f:46073a

[EL] R. Exel and M. Laca, Partial Dynamical Systems and the KMS Condition, preprint (2000).

[EFW] M. Enomoto, M. Fujii and Y. Watatani, KMS states for gauge action on $\mathcal{O}_{A}$. Math. Japon. 29 (1984), no. 4, 607-619. MR 86b:46110

[Eva] D.E. Evans, On $\mathcal{O}_{n}$, Publ. Res. Inst. Math. Sci. 16 (1980), 915-927. MR 82g:46099

[Izu] M. Izumi, Subalgebras of infinite $C^{*}$-algebras with finite Watatani indices. I. Cuntz algebras, Comm. Math. Phys. 155 (1993), no. 1, 157-182. MR 94e:46104

[Kit] B.P. Kitchens, Symbolic dynamics. One-sided, two-sided and countable state Markov shifts, Universitext. Springer-Verlag, Berlin, 1998. MR 98k:58079

[Oka] R. Okayasu, Cuntz-Krieger-Pimsner algebras associated with amalgamated free product groups, Publ. Res. Inst. Math. Sci. 38 (2002), no. 1, 147-190.

[OP] D. Olesen and G.K. Pedersen, Some $C^{*}$-dynamical systems with a single KMS state, Math. Scand. 42 (1978), no. 1, 111-118. MR 80a:46041

[RR] J. Ramagge and G. Robertson, Factors from trees, Proc. Amer. Math. Soc. 125 (1997), no. 7, 2051-2055. MR 97i:46108

[Spi] J. Spielberg, Free product groups, Cuntz-Krieger algebras, and covariant maps, Internat. J. Math. 2 (1991) 457-476. MR 92j:46120

[SV] S. Strătilă, and D. Voiculescu, Representations of AF-algebras and of the group $U(\infty)$, Lecture Notes in Mathematics, Vol. 486. Springer-Verlag, Berlin-New York, 1975. viii+169 pp. MR 56:16391

Department of Mathematics, Kyoto University, Kyoto 606-8502, Japan

E-mail address: rui@kusm.kyoto-u.ac.jp 